\title{
Full Polarimetric Millimetre Wave Radar for Stand-off Security Screening
}

\author{
Eddie Blackhurst ${ }^{\mathrm{a}}$, Neil Salmon ${ }^{\mathrm{b}}$, Matthew Southgate ${ }^{\mathrm{b}},{ }^{\mathrm{a}}$ School of Physics and Astronomy, The \\ University of Manchester, Jodrell Bank, SK11 9DL. ' Faculty of Science and Engineering, \\ Manchester Metropolitan University, All Saints, Oxford Road, M15 6BH.
}

\begin{abstract}
The development and measurements are described of a frequency modulated continuous wave (FMCW) mono-static millimetre wave full polarimetric radar, operating at k-band (18 to $26 \mathrm{GHz})$. The system has been designed to explore the feasibility of using full polarimetry for the detection of concealed weapons, and person borne improvised explosive devices (PBIED). The philosophy of this scheme is a means to extract the maximum information content from a target which is normally in the single spatial pixel (sometimes sub-pixel) configuration in stand-off (tens of metres) and crowd surveillance scenarios. The radar comprises a vector network analyser (VNA), an orthomode transducer and a conical horn antenna. A calibration strategy is discussed and demonstrated using a variety of known calibration targets with known reflective properties, including a flat metal plate, dihedral reflector, metal sphere, helix and dipole. The orthomode transducer is based on a high performance linear polarizer of the turnstile type with isolation better than $35 \mathrm{~dB}$ between orthogonal polarisations. The calibration enables the polarimetric Sinclair scattering matrix to be measured at each frequency for coherent polarimetry, and this can be extended using multiple measurements via the Kennaugh matrix to investigate incoherent full polarimetry.
\end{abstract}

Keywords: Concealed weapons detection, FMCW radar, Mono-static radar, full polarimetry, millimetre wave radar, non-imaging sensor, Sinclair matrix, security screening.

\section{INTRODUCTION}

In a stand-off security screening scenario potential threats concealed under clothing are likely to have an angular size smaller than the diffraction limited beam size $(\lambda / \mathrm{D})$, for a sensor aperture size $\mathrm{D}$. As an imaging sensor this constitutes essentially a single pixel and therefore the scope for extracting spatial information in the usual way via image processing is rather limited. As a result interest turns to other dimensions whereby information from a single spatial pixel may be extracted. Depth (or range) resolution (by increasing radio frequency bandwidth) and polarimetry (by using more sophisticated transceivers) are two options whereby more information about a target can be accumulated.

The benefits of millimetre wave polarimetric sensing for concealed weapons detection on persons has been examined by a number of researchers [1], [2]. The potential for portability of such sensors has been explored for the screening of people in crowds for concealed metal and non-metallic threats, such as metal and ceramic knives and guns and person borne improvised explosive devices (PBIED)[3],[4],[5].

Extending the capability to one of full polarimetry, by measuring the phase and amplitude of all combinations of co and cross-polar responses enables the full polarimetric Sinclair scattering matrix (a $2 \times 2$ complex matrix) to be populated. This area has been extensively researched and reported on for remote sensing applications, typically for sensors based on aircraft and satellite [6][7][8][9], so has been ripe to be exploited by the security sensing community. Full polarimetry for security screening was initially investigated in [10], [11] using a pseudo monostatic (transmitter and receiver colocated). This used adjacent transmitter and receiver antennas and ran a number of decomposition algorithms on experimental data.

The work presented here is concerned with the development of a true monostatic full polarimetric measurement capability, by the use of an orthomode transducer (OMT)[13]. The paper presents performance measurements made on the OMT, describes the full polarimetric monostatic radar configuration and presents initial measurement at close range, 
typically one or two metres, and their interpretation. Interpretation will take the form of comparing measured Sinclair matrices with those expected from the theory for a variety of targets, such as plane, dihedral, trihedral and helical structures. Understanding of signatures should then enable the technique to be refined, extending it to longer ranges.

\section{FULL POLARIMETRY}

A simple example of how polarimetry can be useful in a security screening scenario can be made by considering the single bounce reflection from the human body. Single bounce reflections from the human torso or a smooth flat conductive surface tend to cause co-polar reflection, (VV) and (HH). Objects that can cause multiple reflections such as metal targets with sharp edges and angular features, an example being a PBIED with embedded shrapnel tend, to produce strong cross polar reflection (HV) and $(\mathrm{VH})$, as reported by [3][4][5]. Furthermore, by following techniques from the remote sensing radar community there are techniques to determine whether reflections are a result of odd or even numbers of reflections [9].

Making full polarimetric radar measurements is concerned with stimulating a target with one polarisation and measuring the phase and amplitude response in both co- and cross-polar returns, before stimulating the target in the orthogonal polarisation and measuring both co and cross-polar response. In doing this a $2 \times 2$ (complex) polarimetric scattering matrix is measured, commonly referred to at the Sinclair (back) scatter matrix, as indicated in Eq. 1.

$$
\mathrm{S}=\left[\begin{array}{ll}
S_{11} & S_{12} \\
S_{21} & S_{22}
\end{array}\right]=\left[\begin{array}{ll}
S_{H H} & S_{H V} \\
S_{V H} & S_{V V}
\end{array}\right]
$$

If measurements are made using two orthogonal linear polarisations, the system is said to measure in the linear polarisation basis. If measurements are made using two orthogonal circularly polarised waves (right hand and left hand) the system measures in the circular polarisation basis. Measuring in the linear basis generates the Sinclair matrix as indicated in Eq.1.

There are an infinite number of orthogonal polarisation bases and data recorded in one basis can be converted to another basis using a unitary similarity transformation [8]. This means any basis can be used, but experimentally the linear or circular are the easiest to generate with precision using standard transmission line components and waveguides. Using this transformation [9], converting from the measurements made in the linear basis into the circular basis can be performed using Eq. 2.

$$
\left[\begin{array}{ll}
S_{R R} & S_{R L} \\
S_{L R} & S_{L L}
\end{array}\right]=\frac{1}{2}\left[\begin{array}{cc}
S_{H H}-S_{V V}-2 j S_{H V} & -j\left(S_{H H}+S_{V V}\right) \\
-j\left(S_{H H}+S_{V V}\right) & S_{V V}-S_{H H}-2 j S_{H V}
\end{array}\right]
$$

Full polarimetry from the field of optics (where historically only intensity could be measured) full polarimetry is defined as measuring the response indicated by the Stokes parameters (a four element vector fully defining the polarimetric state of radiation), to a simulation of the target in all possible combinations of polarimetric excitation. This generates a $4 \times 4$ real matrix, referred to in a backscatter configuration as the Kennaugh matrix, or in the forward direction as the Mueller matrix [12]. Using intensities to measure the Stokes parameters is referred to as an incoherent method, as phase of the optical wave is not measured.

The four Stokes parameters correspond to: 1) radiation intensity, 2) degree to which radiation is horizontally polarised (as opposed to vertically), 3) degree to which radiation is linearly polarised at $45^{\circ}$ to the horizontal (as opposed to $-45^{\circ}$ ), 4) degree to which the radiation is right-hand circularly polarised (as opposed to left-hand circularly polarised). Examples of measuring these in a passive system in the millimetre wave band are given in [14].

The essential difference between the Sinclair matrix and the Kennaugh matrix is that the former is measured using coherent radiation and the latter measured using incoherent radiation. As such the former can characterise the response to unpolarised radiation and measure the level of depolarisation of polarised states of radiation. As a means to derive a Kennaugh matrix from the Sinclair matrix it may be possible to average multiple measurements of the Sinclair matrix taken during the depolarisation of coherent waves by a target and thereby create a coherency matrix [8]. This under certain conditions may be converted to the Kennaugh matrix, as measured by incoherent optical methods.

A monostatic measurement configuration is one which lends itself readily to the stand-off security screening scenario. Here the transmitter and receiver are co-located, as opposed to a bistatic configuration, where the two are at separate 
locations. Under these conditions the Sinclair matrix become complex symmetric (ie symmetrical about the leading diagonal), so contains only six independent numbers. Also under a monostatic configuration redundancy is introduced into the Kennaugh matrix, reducing it to a total of nine independent real numbers.

One of the key issues in polarimetry is something referred to as depolarisation. In this context depolarisation is a change in the target which causes a change in the Sinclair matrix. It is an assumption by the remote sensing community that depolarisation is caused by physical movement, of the target or the radar or both. Part of the work will be to determine if, how and why depolarisation occurs in the monostatic security screening scenario.

Understanding the level of depolarisation is important, as if there is no depolarisation, or if depolarisation can be minimised below a certain level, it is possible to use the techniques of coherent processing to analyse data. If there are considerable levels of depolarisation, a wave coherency matrix approach needs to be taken, whereby multiple samples of the Sinclair matrix need to be processed.

The Sinclair matrix when measured can be measured at single frequency, or at a range of frequencies. The measurements can be calibrated using measurements of background and the response from a known target. Fourier transforming the calibrated measurements from the frequency domain, into the time domain, creates the delta function response. This effectively is the response of bandlimited pulse having a length, the inverse of the frequency bandwidth.

\section{METHOD}

The radar presented in this paper is of the monostatic non-imaging type, based around Sinclair matrices generated from measurements made using a Keysite PNAX VNA, with a swept frequency range covering 18 to $26 \mathrm{GHz}$ (K-band). The radar is of the frequency modulated continuous wave (FMCW) type. Figure 1 shows a block diagram of the radar, indicating the key elements: the VNA, the orthomode transducer (OMT) and the corrugate conical horn antenna. Figure 2 shows photographs of the experimental set up, indicating the layout with VNA, the OMT and horn antenna. The OMT is of the linear polariser turnstile type and is of 'split block' construction, the internal architecture is shown in Figure 3 [13].

The system exploits the two ports of the VNA to send out and measure separately the return from two coherent waves. The OMT is arranged to combine orthogonally these two waves in a single waveguide, so that one becomes the horizontal polarisation and the other the vertical linear polarisation. The system thus constitutes a true monostatic measurement system, set to measure in the orthogonal linear polarisation basis. This enables horizontal polarisation to be transmitted to the target and simultaneous measurements to be made on both the horizontal and vertically polarised returns. The VNA calculates the S-parameters in the standard way, so with the OMT on the frontend, these parameters constitute directly the elements of the Sinclair back scatter matrix.

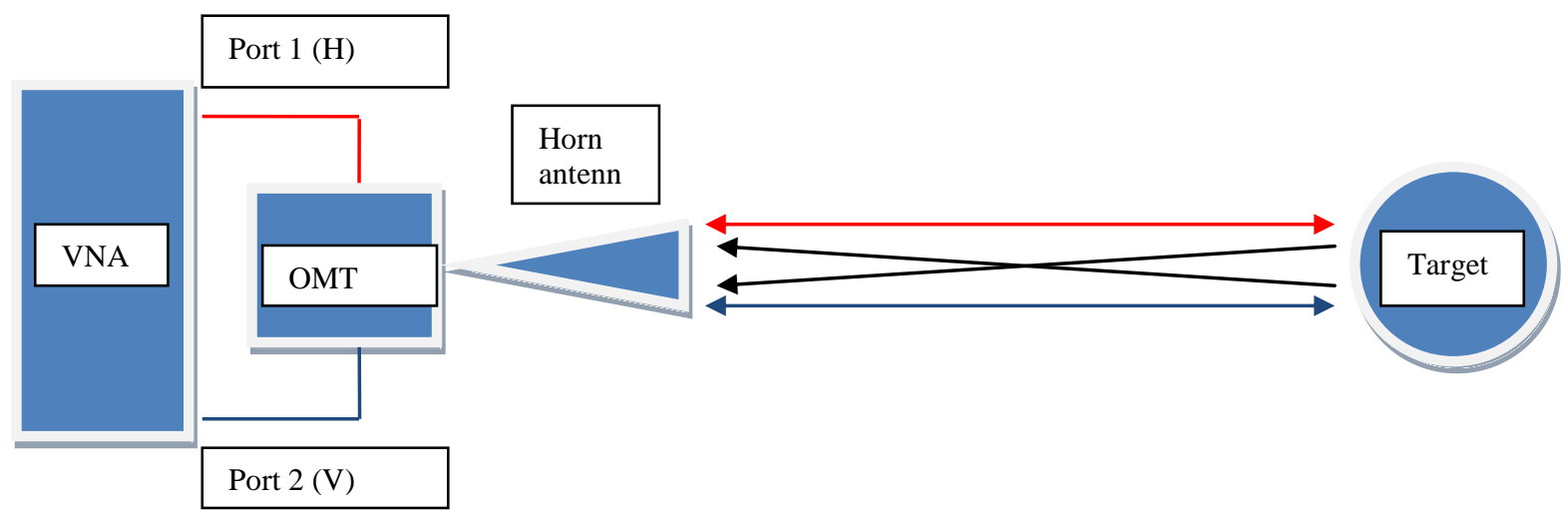

Figure 1. A schematic of the full polarimetric monostatic FMCW radar system 


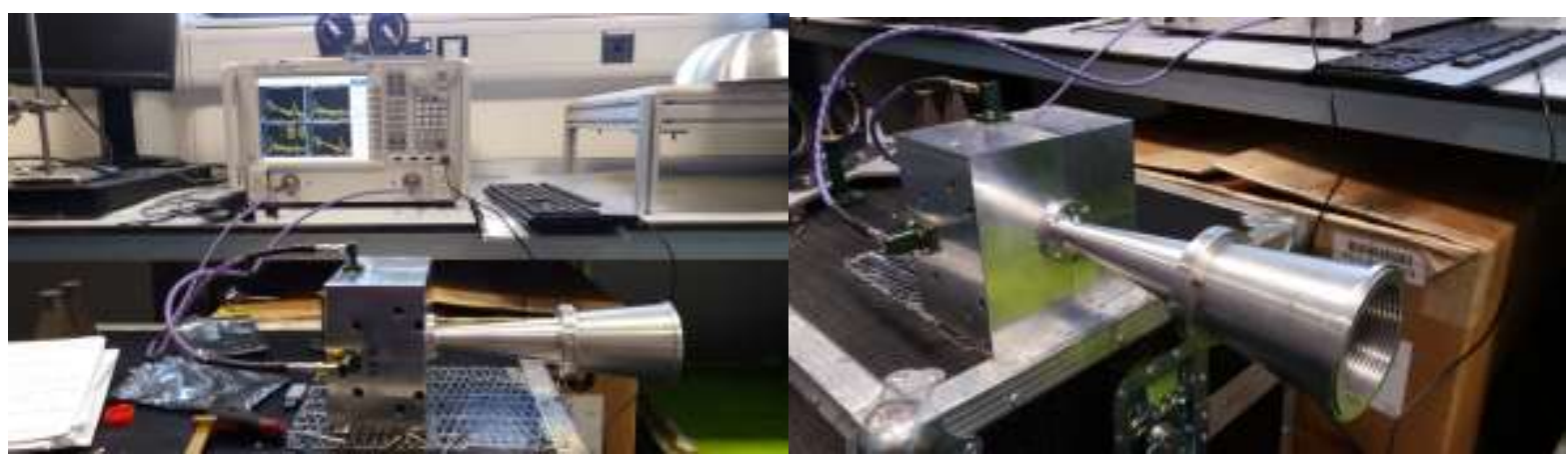

Figure 2. A photograph of the full polarimetric monostatic FMCW radar (left) and a close up of the OMT and conical scalar horn antenna (right).

The internal architecture of the OMT and the turnstile junction can be seen in Figure 3. It comprises four single mode waveguides with a single circular waveguide port at the intersection, the circular waveguide being connected to the conical horn antenna. At the base of the circular waveguide can be seen a rectangular block used as a tuning stub to optimise return loss over a broad band of radiation frequencies. The circular waveguide supports propagation of two fundamental TE11 modes (Pol1 and Pol2 in the figure) as two independent orthogonal linear polarisation states. In receive mode the junction splits Poll equally between rectangular waveguides Polla and Pollb with a $180^{\circ}$ phase shift between them, with no coupling to $2 \mathrm{a}$ or $2 \mathrm{~b}$. Conversely Pol2 is split equally between Pol2a and Pol2b. Signals exiting the turnstile junction are recombined in an E-plane power combiner and exit the rectangular waveguide via a tapered section which matches the standard WR42 (WG20) waveguide size.
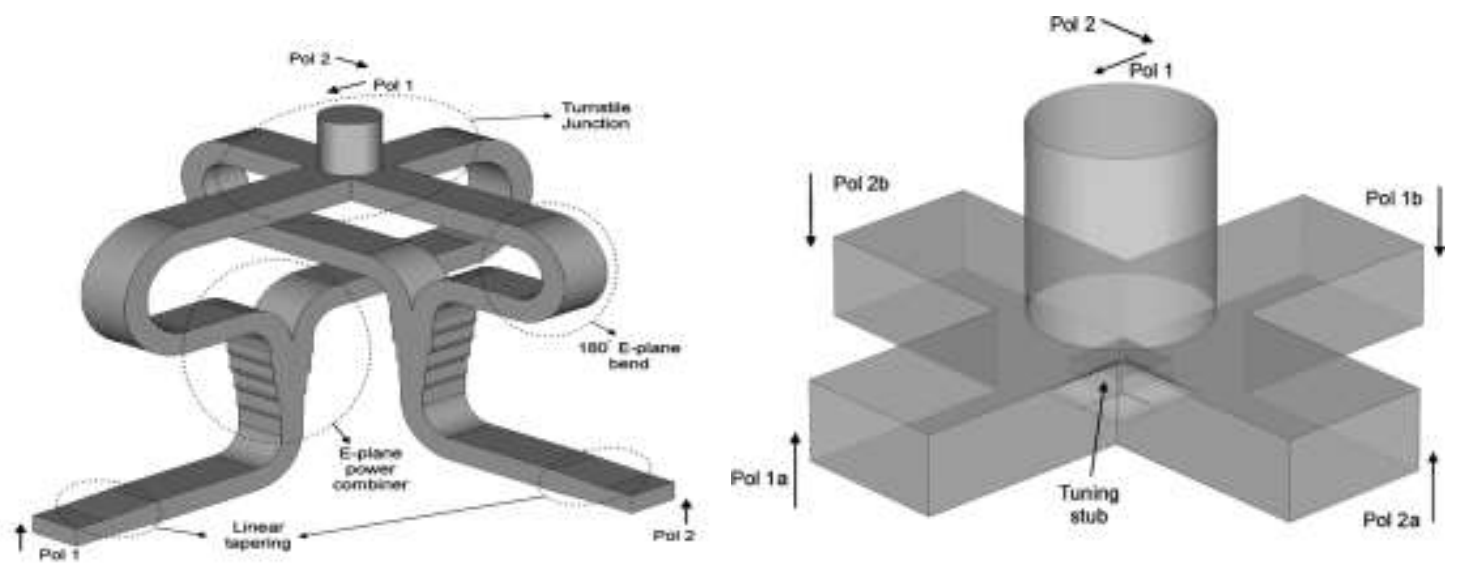

Figure 3. The internal waveguide structure of the OMT (left) and a more detailed illustration of the turnstile junction and E-plane power combiners (right). [13]

The radar is designed around the operation of the VNA, and many of the specifications arise from the settings on this and are summarised in Table 1. The frequency is swept from $18 \mathrm{GHz}$ to $26 \mathrm{GHz}$ and this bandwidth sweep of $8 \mathrm{GHz}$, gives the system its unambiguous range resolution of $\mathrm{c} / 2 \mathrm{~B}$ of $1.875 \mathrm{~cm}$. In the frequency sweeping process, each data point is an average of 10 samples and the complete spectrum comprises a total of 801 points. The frequency sweep time is $99 \mathrm{~ms}$ and the video bandwidth is set to $10 \mathrm{kHz}$. 
Table 1 Radar specification.

\begin{tabular}{|l|c|}
\hline Radar Type & FMCW \\
\hline Frequency Range & 18 to $26 \mathrm{GHz}$ \\
\hline Number of points in spectrum & 801 \\
\hline $\begin{array}{l}\text { Number of samples per spectral } \\
\text { point }\end{array}$ & 10 \\
\hline Transmit power & $0 \mathrm{dBm}(1 \mathrm{~mW})$ \\
\hline Range resolution & $1.875 \mathrm{~cm}$ \\
\hline Sweep time & $98.859 \mathrm{mS}$ \\
\hline Video bandwidth & $10 \mathrm{KHz}$ \\
\hline Antenna gain & $20 \mathrm{dBi}$ \\
\hline Antenna 3dB full beamwidth & $13^{\circ}$ \\
\hline
\end{tabular}

The antenna has a half power full beam width of $13^{\circ}$ with a nominal gain of $20 \mathrm{dBi}$ determined by simulation.

Table 2 Measured polariser r.f characteristics over 18 to $26 \mathrm{GHz}$ (K-Band).

\begin{tabular}{|l|r|r|r|r|}
\hline & $\begin{array}{l}\text { Input Return } \\
\text { Loss }(\mathrm{dB})\end{array}$ & $\begin{array}{l}\text { Worst case Input } \\
\text { Return Loss }(\mathrm{dB})\end{array}$ & $\begin{array}{l}\text { Transmission } \\
\text { Loss (dB) }\end{array}$ & $\begin{array}{l}\text { Polarisation (Isolation) } \\
(\mathrm{dB})\end{array}$ \\
\hline $\mathrm{CH} 1$ & -22.87 & -15.49 & -0.3 & -37.04 \\
\hline $\mathrm{CH} 2$ & -23.08 & -16.72 & -0.26 & -37.05 \\
\hline Circular Port & -23.11 & -15.29 & & \\
\hline
\end{tabular}

\section{CALIBRATION}

An internal calibration of the VNA is required to account for reflections, leakage and cross coupling between components inside the VNA. This is performed using a full twelve term standard through, reflect, line (TRL) calibration, using a set of calibration standards comprising: a quarter-wavelength waveguide section, a couple of waveguide short circuits and a couple of $50 \Omega$ loads.

Since it is the response of that in front of the antenna which is to be determined and the OMT and the horn antenna introduce phase errors, these effects need to be removed. Furthermore, the beam is generally larger than the target, so there will be background reflections from this much wider field. Compensating for these is a two stage process. Firstly the target is measured followed by the scene without the target. The former is subtracted from the latter to get the response of the target alone. Secondly the response of an object with no dispersion is measured. The spectral response of the target is then divided by the spectral response of the dispersionless object. This removes the phase effects in the OMT and the horn antenna, so that which remains belongs only to the target. It also has the effect of zeroing the phase at the location in space where the dispersionless object was placed.

When the background and phase effects of all but the target have been removed, that which remains is the calibrated Sinclair matrix of the target alone. Given the configuration in Figure 1, this means that the standard s-parameters of the network (ie that which is in front of the horn antenna) becomes the Sinclair matrix of the target measured in the linear polarisation basis, as indicated in Eq. 1. The above calibration is made in the frequency domain, so there are a whole series of nominally different Sinclair matrices.

Interpretation of the Sinclair matrices it is sometimes easier in the time domain, as data can be thought of as the propagation of a pulse to and back from the target. The time domain form of the Sinclair matrix can and be made by taking the Fourier transform of the frequency representation. By plotting the complex square of the time domain form of the Sinclair matrix, the dispersion effects of a bandwidth limited pulse, equivalent to that of the frequency sweep, can be 
assimilated. The result of the above calibration in the time domain has the effect of making the zero time delay correspond to the physical location of the dispersionless object. The experimental data from this work is plotted in the results section of the paper in the time domain. This is for ease of interpretation and data validation.

\section{RESULTS}

Measured Sinclair matrices were generated for the following calibration targets;

1. Flat metal plate.

2. Dihedral reflector angled at $45^{\circ}$ between orthogonal polarisations.

3. A metal sphere

4. A long horizontal wire (referred to as the dipole)

5. Helical coils

6. Wall of microwave absorber.

To remove unwanted internal reflections produced by the horn and polarizer, the Sinclair matrices for all targets including both the metal plate and dihedral calibration reflectors, are subtracted from a reference background matrix measured from a wall of microwave absorber.

The linear response of the radar has its co-polar calibration performed using a flat metal plate, a dispersionless object. The metal plate produces a strong co-polar reflection when illuminated with linearly polarized radiation. The cross polar calibration is performed using a dihedral reflector oriented at an angle of $45^{\circ}$; the dihedral reflector produces a strong cross polar reflection and is a dispersionless object. The circular response of the radar has its co-polar response calibrated by the dihedral reflector. The dihedral reflector produces a strong co-polar reflection in this instance. The cross polar response being calibrated using the flat metal plate. This reflector produces a strong cross polar reflection to circularly polarized radiation.

Accurate alignment of targets, in particular the dihedral in the beam of the radar is essential to avoid significant errors in the measured response [15], [16]. Alignments of all targets were performed using a laser pointer and tape measure. Figures 4 show linear basis and circular basis Sinclair matrices (in the time domain, laid out as in Eq. 1 and Eq. 2) for a metal plate target. These measurements confirm the expected result that a flat metal plate produces no change in the orientation of linearly polarised radiation. However, measurements confirm the expected result that right-hand circular polarisation is converted to left-hand polarisation upon reflection from a plane metal surface.
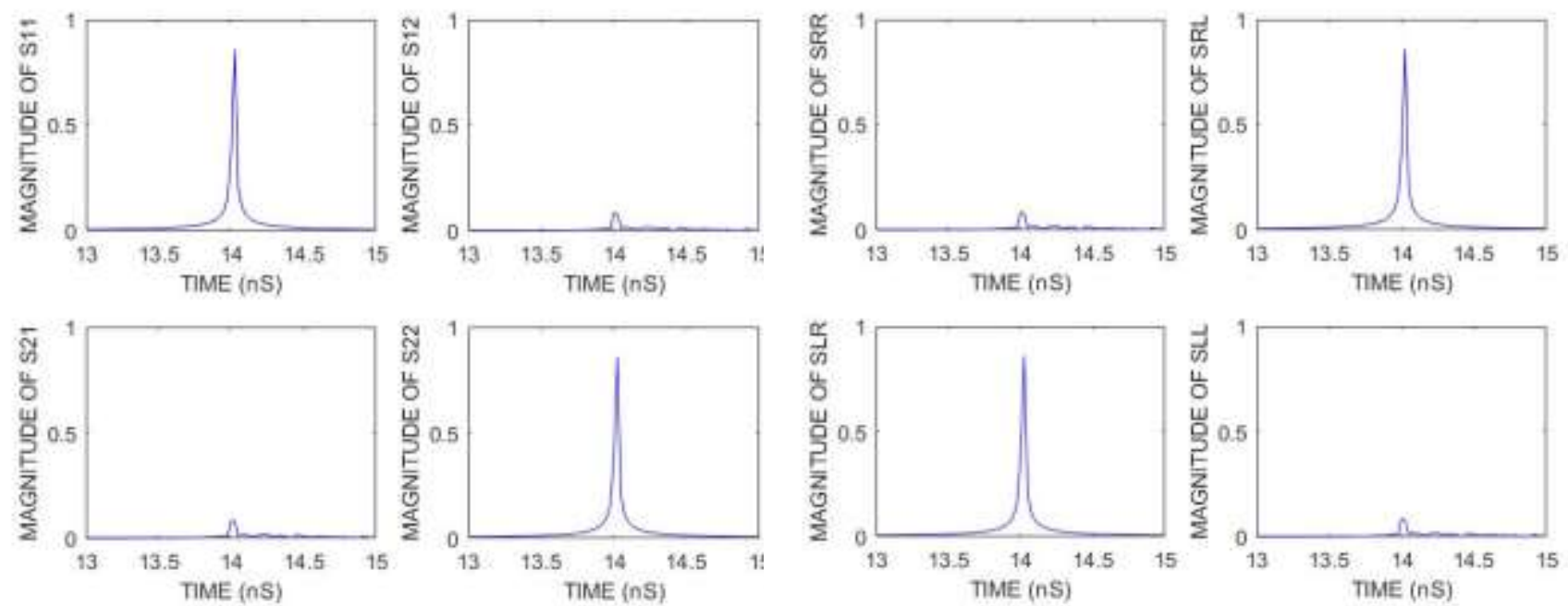

Figure 4. Measured linear polarisation basis Sinclair matrix (left) for a flat metal plate and conversion to circular polarisation basis (right). 
The Sinclair matrices for the dihedral with its ridge located at $45^{\circ}$ to the horizontal as indicated in Figure 5 are shown in Figure 6, for the measured linear polarisation basis, and the derived circular polarisation basis. Confirmation is that linear polarisation is flipped from horizontal to vertical, but that the handedness of circular polarisation is unchanged upon reflection. This is what would be expected from the double bounce reflection from a dihedral in this orientation [9].

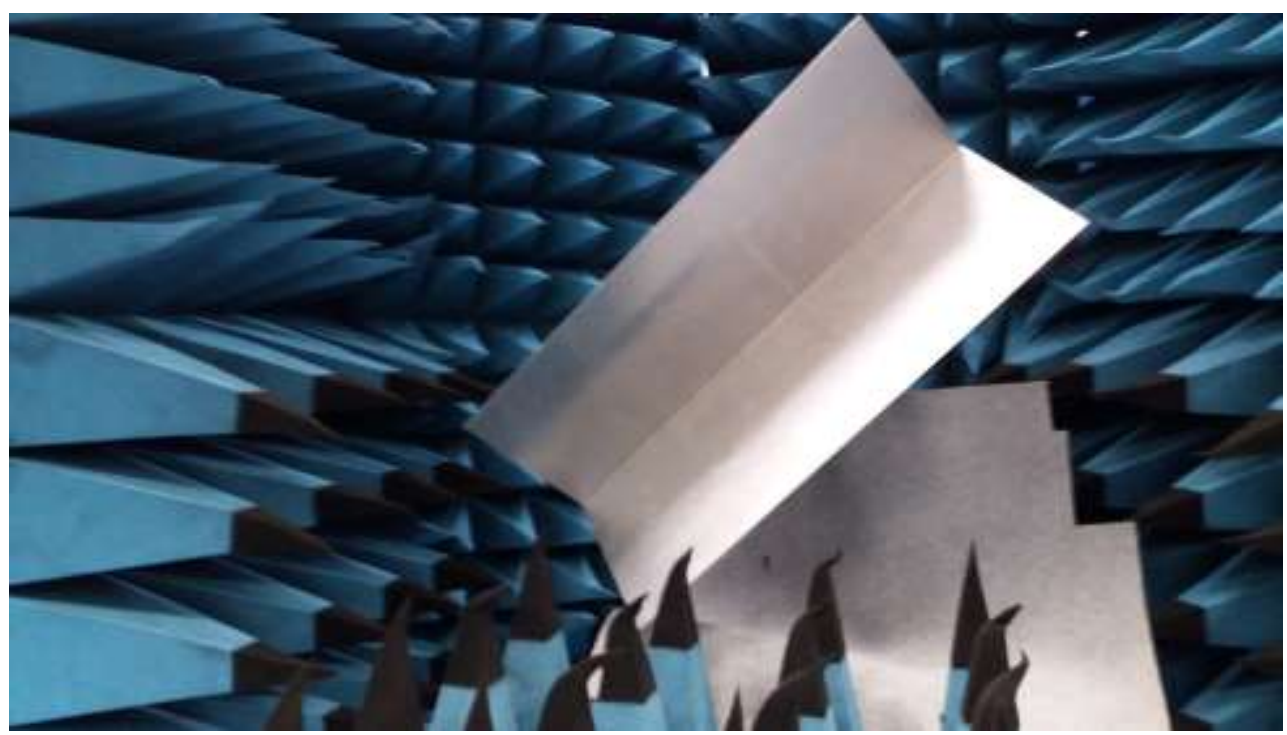

Figure 5. The dihedral reflector orientated at an angle of $45^{\circ}$ to the horizontal
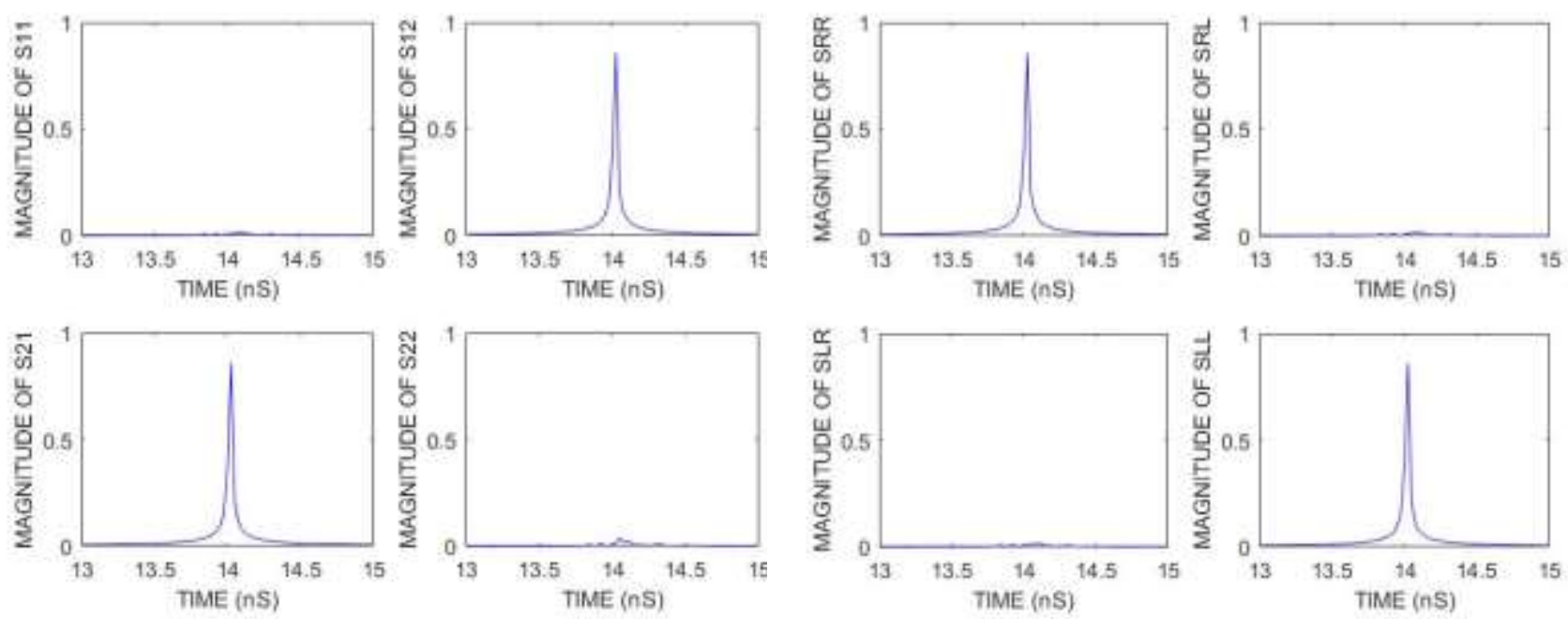

Figure 6. Measured linear polarisation basis Sinclair matrix (left) for a dihedral reflector orientated at an angle of $45^{\circ}$ and conversion to circular polarisation basis (right). 
An $86 \mathrm{~mm}$ diameter stainless steel sphere as a target is suspended in the beam of the radar, as illustrated in Figure 7. The Sinclair matrices from this, shown in Figure 8, show a strong co-polar response and a much smaller cross-polar response, as would be expected from a single bounce reflection [9]. The sphere has depth and there is evidence that the waves travel around the sphere, with part of the radiation being delayed by up to $1 \mathrm{~ns}$. This is consistent with the creeping wave response [17], whereby some portion of the radiation travels around the sphere, before being re-radiated back towards the transmit/receive antenna.

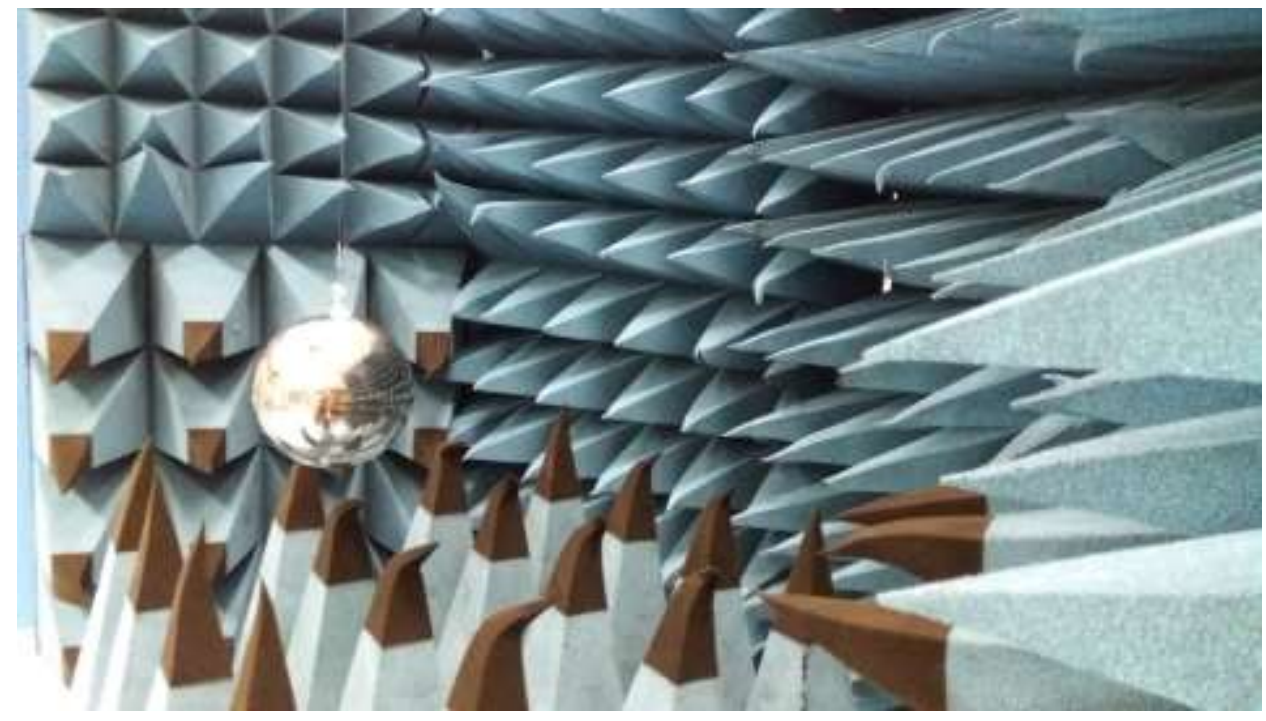

Figure 7. The $86 \mathrm{~mm}$ diameter stainless steel sphere suspended on a thin thread of nylon
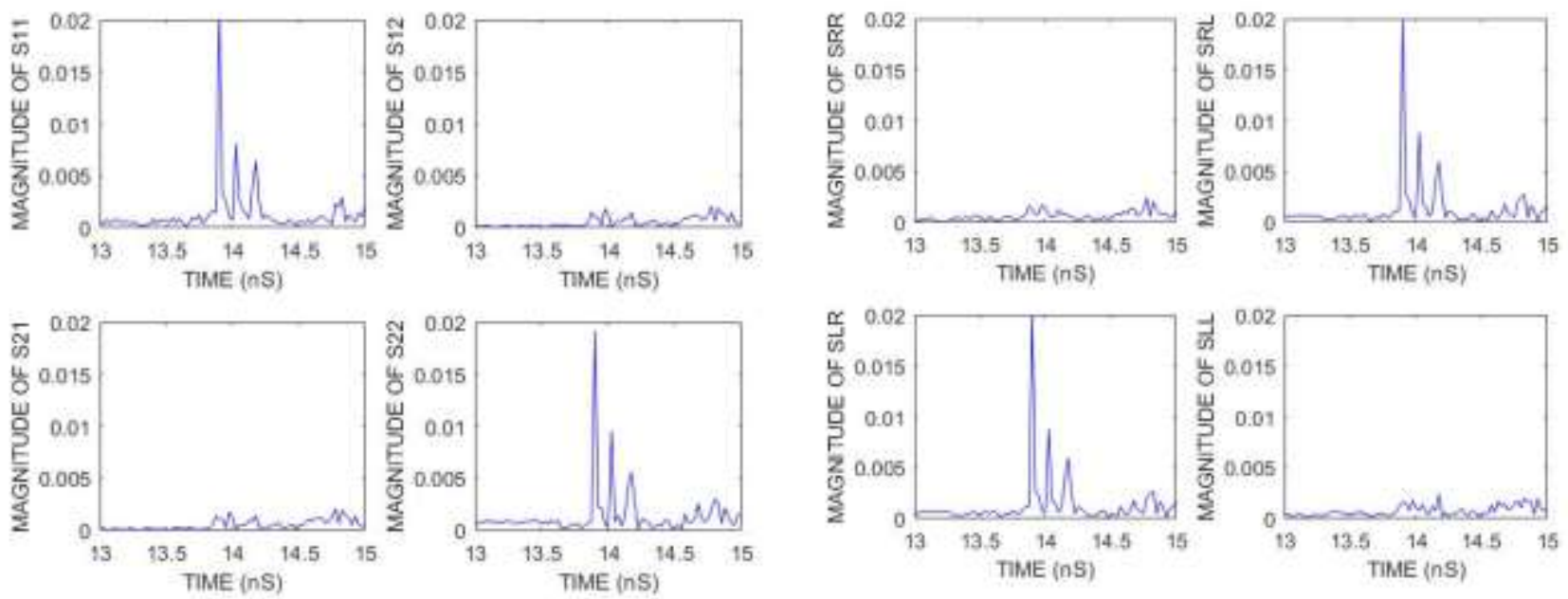

Figure 8. Measured linear polarisation basis Sinclair matrix (left) of the stainless steel sphere and conversion to circular polarisation basis (right). 
The Sinclair matrices for the horizontal dipole (short horizontal wire) of Figure 9 are shown in Figure 10. The wire has a large return in $\mathrm{S}_{\mathrm{HH}}$ and a smaller return in $\mathrm{S}_{\mathrm{Vv}}$. This would be expected, as the wire is much longer in the horizontal direction than its diameter in the vertical direction.

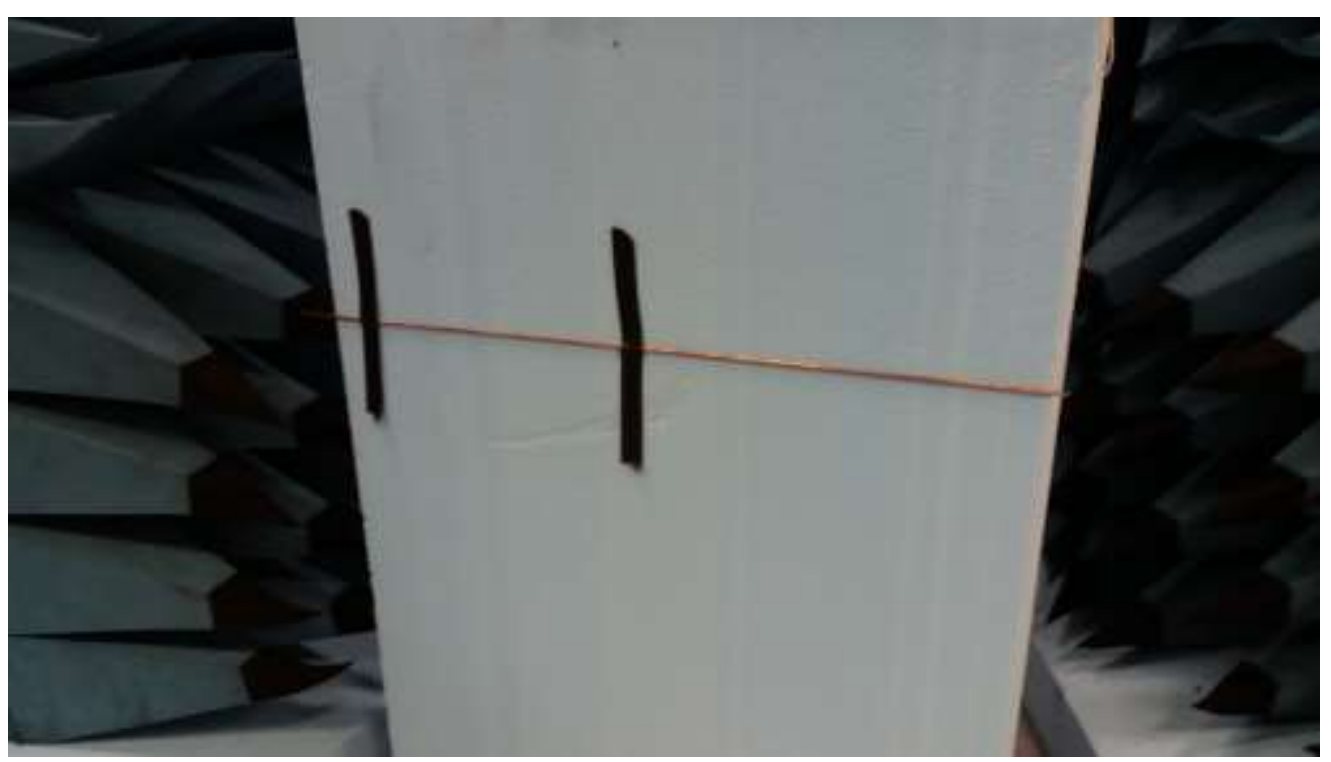

Figure 9. The horizontal wire (dipole).
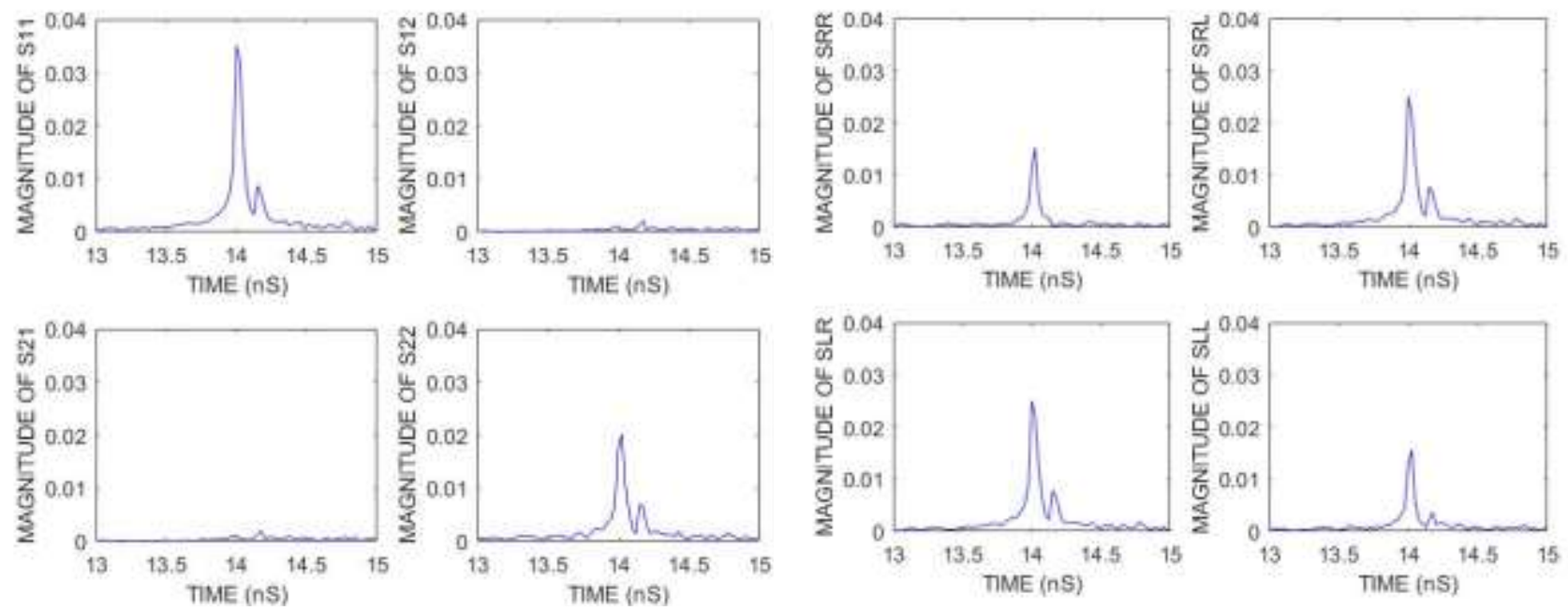

Figure 10. Measured linear polarisation basis Sinclair matrix (left) for a horizontal wire and conversion to circular polarisation basis (right). 
Figure 11 shows ten helical antennas arranged in a hexagonal arrangement in the beam of the radar. All the 5 turn coils are wound in the same direction and are tuned to work at K-band in axial radiation mode. The circular response is shown in Figure 12. Note the largest response occurs in the $S_{\mathrm{RR}}$ component of the Sinclair matrix, as would be expected as this is the wind direction of the helix.

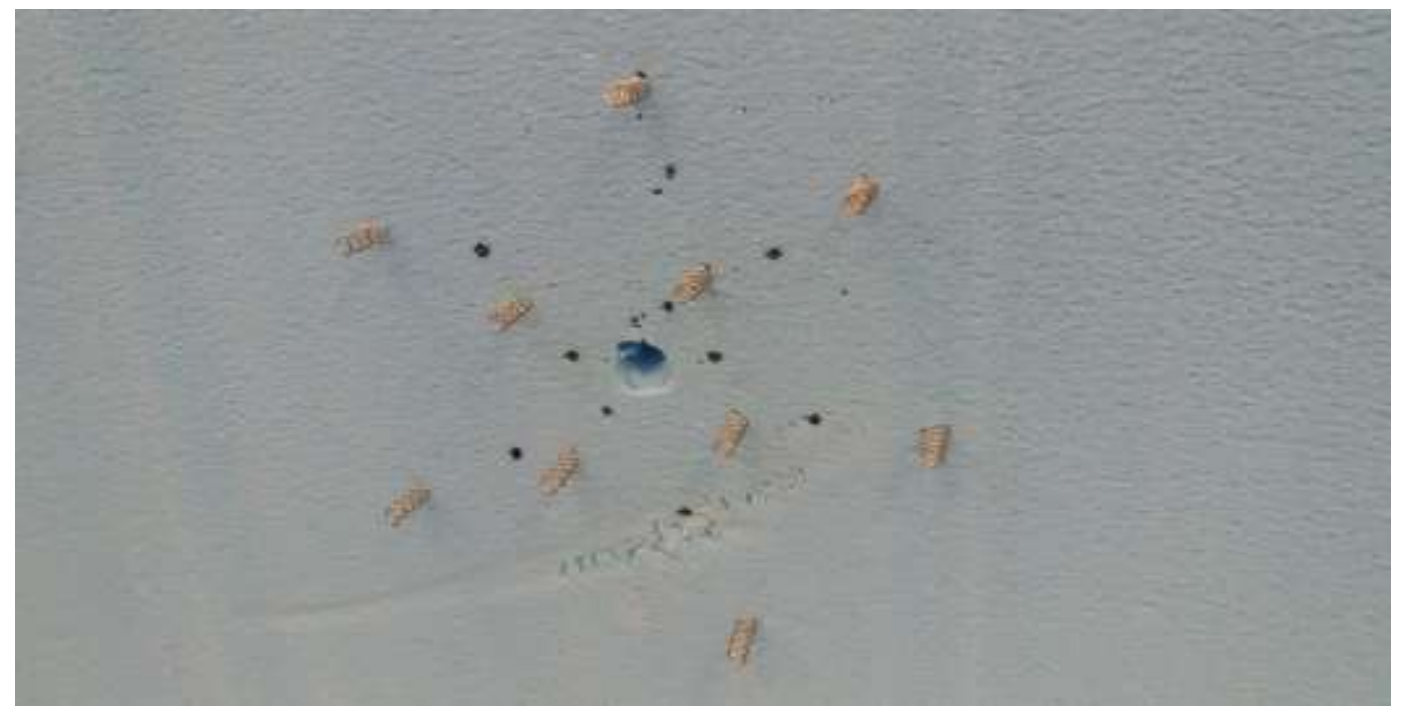

Figure 11. The ten helical antennas.
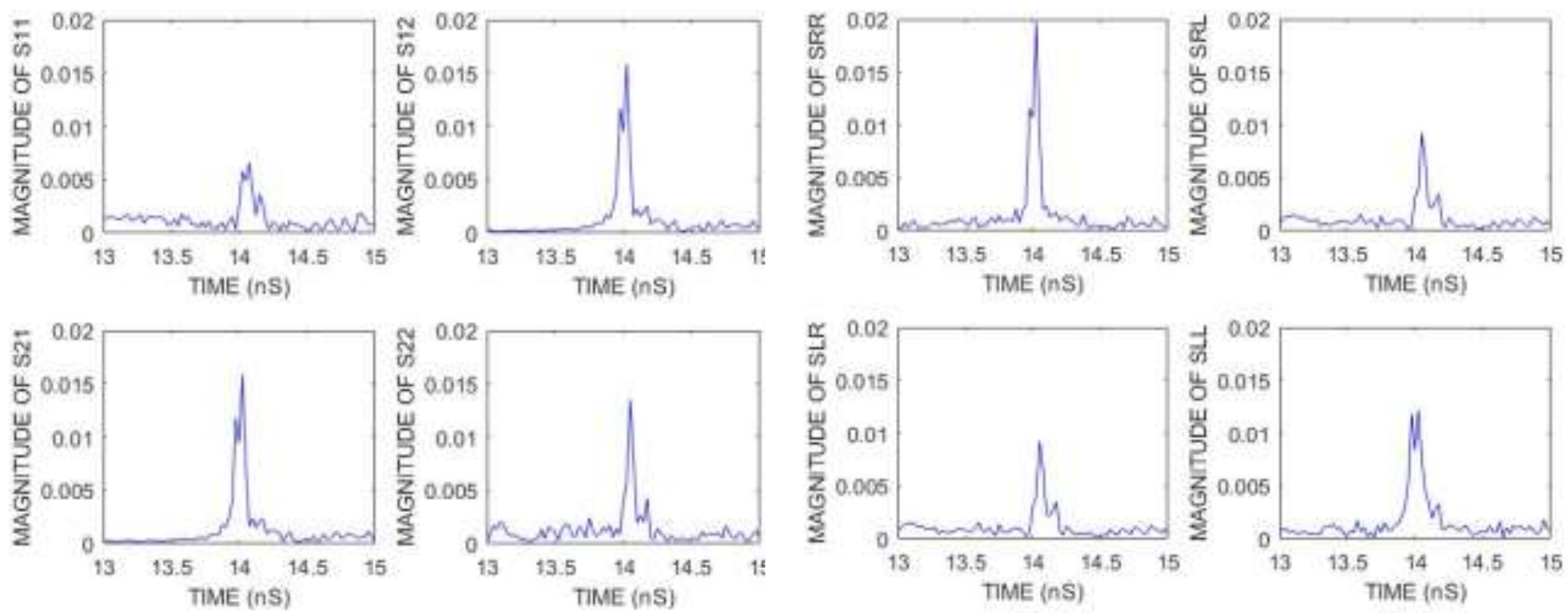

Figure 12. Measured linear polarisation basis Sinclair matrix (left) for the helical antennas and conversion to circular polarisation basis (right).

\section{FUTURE WORK}

The future work will concentrate on identifying the full polarimetric signatures of the human body alone, threat items alone, and when threats are concealed under clothing on the human body. These measurements will be made whilst these objects or any items in the immediate surroundings are not moving, to determine the reproducibility of the Sinclair matrices. If the matrices are perfectly reproducible there is no depolarisation in the system, so coherent decomposition 
algorithms might then be used on the data. Under these conditions it may be possible to determine the coordinates of the Huynen polarisation fork [6] for each target separately as a means to identification. Movement is expected to introduce depolarisation into the system, which results in changes to the Sinclair matrices. Movement of objects could then be made and changes to the matrices will be noted and compared with theory. In the presence of depolarisation incoherent decomposition algorithms will be investigated, which includes creating from the Sinclair matrices the coherency or covariance matrices [8]. In the case of the human body alone it may be useful to investigate the possibility that the skin of the human body has chirality in its response, which would be detected in the response to circular polarisation, as shown in [18].

\section{REFERENCES}

[1] Sheen, D.M., McMakin, D.L., Lechelt, W.M. and Griffin, J.W., "Circularly polarized millimeter-wave imaging for personnel screening," in Passive Millimeter-Wave Imaging Technology VIII, Orlando, (2005).

[2] Essen, H., Fuchs, H.-H., Hägelen, M., Stanko, S., Nötel, D., Erukulla, S., Huck, J., Schlechtweg, M., Tessmann, A. "Concealed Weapon Detection with Active and Passive Millimeter wave Sensors, Two Approaches", German Microwave Conference, GeMiC, Universität Karlsruhe (TH), March 28 - 30, (2006).

[3] Andrews, D.A., Bowring, N., Rezgui, N-D, Southgate, M., Guest, E., Harmer, S., Atiah, A., "A multifaceted active swept millimetre-wave approach to the detection of concealed weapons", Proc. SPIE vol. 7117, Millimetre Wave and Terahertz Sensors and Technology, Cardiff, United Kingdom, (2008).

[4] Harmer, S.W., Bowring, N., Andrews, D., Rezgui, N-D, Southgate, M. and Smith, S. "A Review of Nonimaging Stand-Off Concealed Threat Detection with Millimeter-Wave Radar," IEEE Microwave magazine, vol. 13, no. 1, pp. 160 - 167, ( 2012).

[5] Southgate, M.J. 'Remote Detection of Concealed Guns and Explosives", PhD Thesis, Manchester Metropolitan University, Manchester, UK, (2013).

[6] Börner, W-M., "Introduction to Synthetic Aperture Radar (SAR) Polarimetry", Wexford College Press, 30th December, (2007). ISBN 9781934939062

[7] S. R. Pottier and E. Cloude, "A Review of Target Decomposition Theorems in Radar Polarimetry," IEEE Transactions on Geoscience and Remote Sensing, vol. 34, no. 2, pp. 498-518, (1996).

[8] Cloude, S.R., "Polarisation: Applications in remote sensing", Oxford University Press. (2010).

[9] Massonnet D. and Souyris, J-C., "Imaging with synthetic aperture radar", Engineering Sciences: Electrical Engineering, CRC Press, (2008).

[10] O'Reilly, D., Bowring, N., Rezgui, N-D, and Andrews, D., "Target decomposition and polarimetric radar applied to concealed threat detection," SPIE Millimetre Wave and Terahertz Sensors and Technology VI, vol. 89000D, (2013).

[11] O'Reilly, D. "A feasibility study on the application of polarimetric decomposition algorithms to the detection of concealed weapons", PhD thesis, Manchester Metropolitan University, Manchester, (2016).

[12] Gil, J.J and Ossikovski, "Polarized Light and the Mueller Matrix Approach", CRC press, (2016).

[13] N. Alessandro and P. R. L., "A Turnstile Junction Waveguide Orthomode Transducer," IEEE Trans. MTT, (2006).

[14] Salmon, N.A., Appleby, R., Coward, P., "Polarimetric passive millimetre-wave imaging", DOI: 10.1117/12.438147, SPIE Proc. vol. 4373, Passive Millimeter-Wave Imaging Technology V, Orlando, (2001)

[15] F. T. U. Kamal Sarabandi, "Calibration of Polarimetric Radar Systems with Good Polarisation Isolation," IEEE Trans. on Geoscience and Remote Sensing, vol. 28, no. 1, pp. 70-75, (1990).

[16] R. N. J. v. S. C.M.H. Unal, "Calibration of a Polarimetric Radar Using a Rotatable Dihedral Corner Reflector," IEEE Transactions on Geoscience and Remote Sensing, vol. 32, no. 4, pp. 837-845, (1994).

[17] Cheney, M., \& Borden, B., 'Fundamentals of radar imaging', Siam, (2009).

[18] Hayut, I., Ishai, P.B., Agranat, A.J. and Feldman, Y., "Circular polarization induced by the three-dimensional chiral structure of human sweat ducts", PHYSICAL REVIEW E 89, 042715 (2014). 